\title{
Multiple pleural biopsy with the Abrams needle
}

\author{
I P F MUNGALL, P N COWEN, N T COOKE, T C ROACH, AND N J COOKE
}

From the Departments of Medicine and Pathology, the General Infirmary and University of Leeds, Leeds

ABSTRACT Multiple pleural biopsies (up to 10) were taken with the Abrams needle througs a single aspiration site in 55 patients with pleural effusions. A definite positive or suggestive pleur能 biopsy diagnosis was made in 33 patients $(60 \%)$. In 32 of the 33 patients the pathological abnorma lity was confined to only a proportion of the biopsies. Pleural biopsies were positive or suggestives in 26 out of 36 patients $(72 \%)$ eventually shown to have tumour and seven out of eight patients $(88 \%$ ) with tuberculosis. Small pneumothoraces occurred in four patients and surgical emphysem in two.

The standard method of using the Abrams pleural biopsy "punch" is to take one biopsy during a single aspiration and if this is negative to repeat the procedure later at a different site. ${ }^{1-3}$ Lowell ${ }^{4}$ has advocated multiple biopsies, up to three through the same site, on the basis that this may improve the likelihood of a histological diagnosis, but this has not been tested in a clinical trial. The present study was designed to assess prospectively the value of multiple pleural biopsy during a single aspiration procedure.

\section{Methods}

Fifty-five patients ( 29 men, 26 women, age range 26-90 years) presenting consecutively with pleural effusions to a district general and a specialist chest hospital were studied. Pleural biopsies were obtained with an Abrams needle by four operators only, using an identical technique. Initially fluid for cytology was withdrawn through a venepuncture needle. Usually five biopsies (range two to 10) were then taken through the same puncture site, the Abrams needle being withdrawn each time. Successive biopsies were made in slightly different positions radially around the inferior and lateral margins of the puncture site. In patients with thin chest walls a firmly held swab was applied between biopsies to prevent a pneumothorax. The pleural effusion was subsequently aspirated through the Abrams needle. The 271 biopsies were sectioned and stained with haematoxylin and eosin in the standard manner.

Address for reprint requests: Dr N J Cooke, The General Infirmary, Great George Street, Leeds LS1 3EX.
They were sectioned at three levels, except for $\frac{\text { 栗 }}{0}$ biopsies in which only two sections containire pleura could be made. For the purposes of the study all the biopsies were examined in randog order by a single pathologist who was unaware the source of each section. The biopsies weere divided into three categories: (1) histologicali diagnostic, or positive, (2) suggestive of a diag nosis, and (3) negative.

In the first group either easily recognised malignant tumour was seen or tuberculosis dia $\overline{g_{f}}$ nosed. Occasionally the tumour could be classified as squamous, oat cell, or adenocarcinoma bu more often, no more information than "anaplast carcinoma" could be given. Tuberculosis wås diagnosed when typical granulomata wi Langhans' giant cells and caseation were seeñ: All three had to be present but the presence of acid-alcohol-fast bacilli was not a prerequisite fơ this diagnosis. In the case of tumours in the second group, doubt was expressed when atypical cells were seen which could possibly have bee either anaplastic, neoplastic cells or non-neos plastic cells from an inflammatory process-that is, active fibroblasts or reactive mesothelial cells In the case of tuberculosis in the second group granulomata containing epithelioid cells be showing no caseation or typical Langhans' celds resulted in a suggestive report. A negative repo meant that there was nothing at all in the histo logical appearances which might suggest neoplasig or tuberculosis (although non-specific inflanof matory changes may have been present). Cyto. logical specimens were similarly examined and classified by the routine diagnostic service of the Pathology Department. 


\section{Results}

On the basis of all biopsies submitted, a positive histological diagnosis was made in 19 of the 55 patients and a suggestive diagnosis in 14 . Of the 19 patients in the former group, in only one were all the biopsies positive, while in seven some biopsies were negative, in one some biopsies were suggestive, and in 10 patients some biopsies were suggestive and some negative.

Similarly in all 14 patients in the suggestive diagnosis group the pathological abnormality was confined to only a proportion of the biopsies. The distribution of pathologically abnormal biopsies in the two groups is shown in fig 1 . For the positive diagnosis group, in most patients between 30 and $70 \%$ of biopsies were abnormal, the range being $14-100 \%$. For the suggestive diagnosis group the range was $15-54 \%$.

The contribution of pleural biopsy to making a diagnosis is demonstrated in fig 2 . Patients have been divided on the basis of their final diagnosis. Of 36 patients eventually shown to have a carcinoma, $17(47 \%)$ had a positive pleural biopsy diagnosis and $26(72 \%)$ either a positive or suggestive diagnosis. Corresponding figures for the eight patients with tuberculosis were two $(25 \%)$ and seven $(88 \%)$, and for all 55 patients 19 $(35 \%)$ and $33(60 \%)$. Eleven patients were thought to have miscellaneous conditions, namely rheumatoid pleural effusion, sarcoidosis, lymphoblastic leukaemia, pneumonia (two cases),

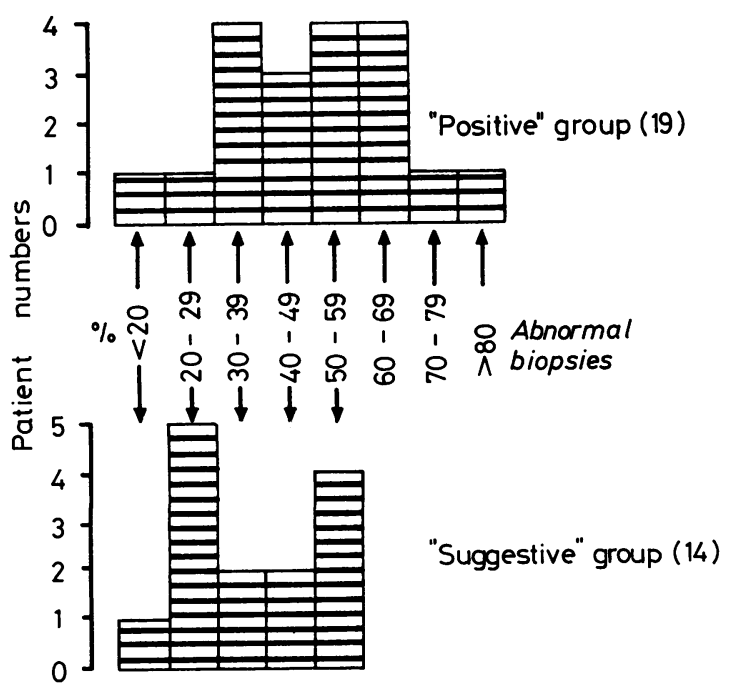

Fig 1 Percentage of pathologically abnormal biopsies in each patient. "Positive" group above; "suggestive" group below.

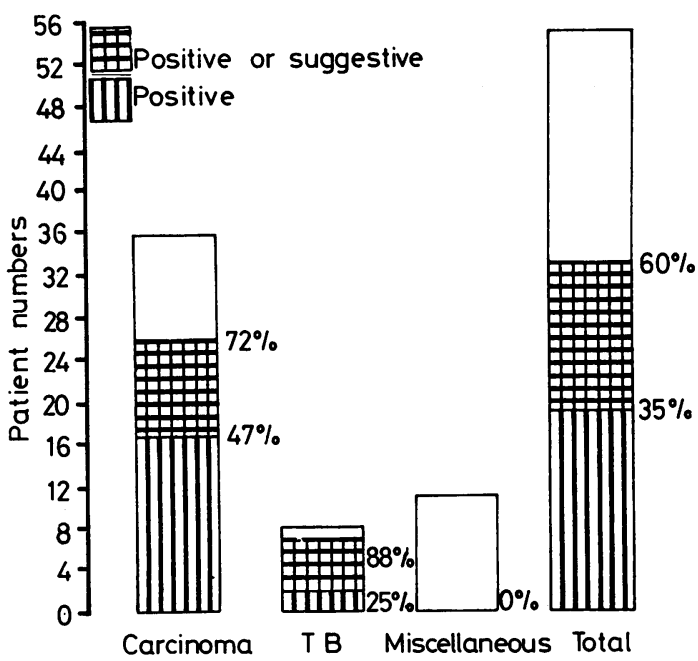

Fig 2 Patients divided on the basis of their final diagnosis: carcinoma, tuberculosis $(T B)$, or miscellaneous conditions. Open columns represent total numbers in each group, vertical hatching those patients with a positive pleural biopsy diagnosis, and squared hatching those patients with a suggestive pleural biopsy diagnosis. The results for all 55 patients are shown in the right hand column.

pulmonary infarction (two cases), possible metastasis from a synovioma, non-tuberculous empyema, congestive cardiac failure, and lymphoedema. Pleural biopsies in all patients in this miscellaneous group were negative.

The only complications were four shallow pneumothoraces and two episodes of surgical emphysema. None of these required active treatment. Malignant cells were detected on fluid cytology in five patients and cells suggestive of malignancy in three. In six of those patients a diagnosis of carcinoma had already been made by pleural biopsy. Thus combined pleural biopsy and fluid cytology provided a positive or suggestive diagnosis in 28 of the 36 patients $(78 \%)$ with malignant effusions.

\section{Discussion}

Multiple pleural biopsy gave a diagnosis in $88 \%$ of patients with tuberculosis and $72 \%$ of patients with carcinoma. In a review of the conventional single pleural biopsy technique with a variety of needles in more than 1200 patients, Schools ${ }^{5}$ reported a diagnostic yield of $70 \%$ for tuberculous effusions and $58 \%$ for malignant effusions, the figures in those series using the Abrams needle being similar at $73 \%$ and $59 \%$ respectively. 
In two more recent series the diagnostic pick-up rate with the Abrams needle has been, for tuberculosis and carcinoma respectively, $71 \%$ and $40 \%{ }^{6}$ and $88 \%$ and $40 \% .{ }^{7}$ In the present series the diagnostic pick-up rate includes patients with both positive and suggestive pleural biopsy diagnosis and as this differentiation was not made in the other series there may not be strict comparability. However, in every case the suggestive diagnosis was used as a working diagnosis and in every case ultimately proved to be correct. One series in which both positive and suggestive pleural biopsy diagnoses have been combined is that of Hampson and Karlish ${ }^{3}$ who reported a diagnostic yield of $75 \%$ in their 32 patients with tuberculosis and $62.5 \%$ in 40 patients with carcinoma.

A similar attempt to provide better biopsy material for pathological examination was made by Moghissi, ${ }^{8}$ who devised a circular cutting needle which takes a biopsy around the whole circumference of the puncture site. The diagnostic pick-up rate in Moghissi's series of 18 patients was excellent with a diagnosis of tuberculosis or carcinoma being made in 15 patients and non-specific inflammation in three. However the needle is cumbersome to use and has not proved popular.

In view of the patchy distribution of pleural tumour repeat biopsies at different sites might be expected to improve the diagnostic yield. Schools ${ }^{5}$ reported positive repeat biopsies in 33 of 127 patients $(26 \%)$ in whom the initial biopsy had been negative. Similar repeat biopsy pick-up rates (30\% and $27 \%$ ) were reported more recently by Scerbo et $a l^{6}$ and Hoff and Li Volsi ${ }^{9}$ respectively. In the present series two patients eventually shown to have carcinoma had repeat biopsies at two different sites but in neither were these helpful. The 10 patients in the carcinoma group with a negative pleural biopsy (fig 2) were eventually diagnosed as having tumour either by bronchoscopy (three cases), thoracoscopy (two cases), pleural fluid cytology (two cases), closed lung biopsy, pericardectomy, or discovery of a primary elsewhere. In all patients with negative pleural biopsies further levels were cut in addition to the three standard levels, but in no case did these provide additional information. In the patient with a tuberculous effusion and a negative biopsy, the final diagnosis was based on a strongly positive Mantoux test, lymphocyte predominance in the pleural fluid, and response to treatment. With the exception of the lymphoblastic leukaemia an the possible secondary synovioma, the patients if the miscellaneous group would all be expected to have had negative or non-specific pleural biopsies

On fluid cytology, malignant cells or celfs suggestive of malignancy were only detected if eight of the 36 patients in the carcinoma group $(22 \%)$, a much lower pick-up rate than the $50 \overrightarrow{\mathrm{st}}$ $60 \%$ reported in most series. ${ }^{7}$ Technical problems or caution on the part of the cytologist may b possible explanations.

In conclusion, multiple pleural biopsy is a safeo modification of an existing technique which mag lead to a diagnosis that might have been misse if only one biopsy had been taken. The delay of repeat biopsies may also be avoided. The diagnostic pick-up rate seems to have been better than in other series using the conventional sing biopsy technique.

We wish to thank the medical staff of the General Infirmary at Leeds and Killingbeck Hospital, Leeds, for referring patients for th study and Miss KA Lebeter for secretariaf assistance.

\section{References}

1 Abrams LD. A pleural-biopsy punch. Lance 1958; 1:30-1.

2 Mestitz P, Purves MJ, Pollard AC. Pleural biopsy in the diagnosis of pleural effusion. A report of 200 cases. Lancet 1958; 2:1349-53.

3 Hampson F, Karlish AJ. Needle biopsy of the pleura in the diagnosis of pleural effusion. $Q \dot{g}$ Med 1961; 30:249-55.

4 Lowell JR. Pleural effusions. A comprehensiv8 review. Baltimore: University Park Press, 197 $\bar{B}$ 68.

5 Schools GS. Needle biopsy of parietal pleura current status. Tex J Med 1963; 59:1056-61.

6 Scerbo J, Keltz H, Stone DJ. A prospective stud. of closed pleural biopsies. JAMA 1971; 218\% $377-80$.

7 Hirsch A, Ruffie P, Nebut M, Bignon Chretien J. Pleural effusion: laboratory tests id 300 cases. Thorax 1979; 34:106-12.

8 Moghissi K. A ney type of pleural biopsy instrü ment. Br Med J 1961; 1:1534.

9 Hoff DD von, Li Volsi V. Diagnostic reliabilitio of needle biopsy of the perietal pleura. A review of 272 biopsies. An J Clin Pathol 1975; 64 200-3. 